\title{
Super-active regions in solar cycle 24
}

\author{
Anqin Chen ${ }^{1,2}$ and Jingxiu Wang ${ }^{2}$ \\ ${ }^{1}$ Key Laboratory of Space Weather, National Center for Space Weather, China Meteorological \\ Administration, Beijing 100081, PR China \\ email: chenanqin@cma.gov.cn \\ ${ }^{2}$ Key Laboratory of Solar Activity of Chinese Academy of Sciences, National Astronomical \\ Observatories, Chinese Academy of Sciences, Beijing 100012, PR China \\ email: wangjx@nao.cas.cn
}

\begin{abstract}
Comparing with solar cycles 21-23, the level of solar activity in the current cycle is very low. So far, there have been only five SARs and $45 \mathrm{X}$ class flares. The monthly smoothed total solar irradiance decreased sharply by $0.09 \%$ from the maximum of cycle 23 to the minima between cycles 23 and 24. In this contribution, we present new studies on SARs in Cycle 24. The SARs in the current cycle have relatively smaller flare index $\left(I_{\text {flare }}\right)$ and composite vector field index $\left(I_{\text {com }}\right)$ comparing with the SARs in cycles 22 and 23 . There is a clearly linear relationship between $I_{\text {flare }}$ and $I_{\text {com }}$. The emphasis of this contribution is put on the similarity and different behaviors of vector magnetic fields of the SARs in the current solar cycle and the previous ones. We try to get a satisfactory account for the general characteristics and relatively lower level of solar flare activity in Cycle 24.
\end{abstract}

Keywords. Sun: activity, Sun: magnetic field, Sun: active regions

\section{Introduction}

The sun is currently at the descending phase of Solar Cycle 24. However, the solar flare activity level is rather low. We are facing a mini-maximum which followed the grand minima of solar cycles 23-24. Is the current trend of solar activity similar to one of the Maunder, Dalton or Gleissberg-Gnevyshev minimum? Some authors suggested that the current cycle is similar to the Gleissberg-Bnevyshev minima (Feynman \& Ruzmaikin 2011; Solanki \& Krivova 2011), Zolotova \& Ponyavin (2014) thought that cycle 23 would be similar to the cycles on the eve of the Dalton and Gleissberg-Gnevyshev minima. As the strong solar activity of each cycle is basically represented by the super-active regions (SARs) in the cycle, it would be of interests to examine the number and characteristics of SARs in Cycle 24, see if in term of SARs the cycle is a particularly weak cycle.

In an earlier study (Chen et al. 2011, Paper I), 45 SARs were selected according to the sunspot area, the soft X-ray flare index, the $10.7 \mathrm{~cm}$ radio peak flux and the decrease of total solar irradiance (TSI) in solar cycles 21-23. These SARs occupied $0.44 \%$ of all the active regions (ARs), but produced $44 \%$ of all the X class X-ray flares during the same time period. On the basis of Paper I, the vector magnetic field characteristics of 14 SARs and eight large and inactive ARs, which were called fallow ARs (FARs), in cycles 22 and 23 were analyzed (Chen \& Wang 2012, Paper II). It was found that the vector magnetic field characteristics of SARs and FARs were significantly different. Most of the SARs had a higher net magnetic flux, a higher total photospheric free magnetic energy density, a longer magnetic neutral line with a steep horizontal magnetic gradient $\left(\geqslant 300 \mathrm{G} \mathrm{Mm}^{-1}\right)$ and a larger area with strong magnetic shear (shear angle $\geqslant 80^{\circ}$ ). However, the values of these magnetic field parameters for the FARs were mostly very small. Based on these 
Table 1. The list of SARs in Solar Cycle 24

\begin{tabular}{lccrrrrr}
\hline NOAA & Lat. Long. & $\begin{array}{c}\text { date on the disk } \\
\text { YYMMDD-MMDD }\end{array}$ & $\begin{array}{c}\text { Flare } \\
\text { index }\end{array}$ & $\begin{array}{c}\text { Maximum area } \\
(\mu \mathrm{h})\end{array}$ & $\begin{array}{c}10.7 \mathrm{~cm} \text { flux } \\
(s . f . u .)\end{array}$ & $\begin{array}{c}\triangle \text { TSI } \\
(\%)\end{array}$ & $\begin{array}{c}\text { CME velocity } \\
\left(\mathrm{km} \mathrm{s}^{-1}\right)\end{array}$ \\
\hline 11429 & N17 L081 & $120303-0315$ & $\mathbf{1 1 . 8 7}$ & $\mathbf{1 2 7 0}$ & $\mathbf{1 2 0 0 0}$ & $-\mathbf{0 . 1 0 9}$ & 2684 \\
11520 & S17 L084 & $120706-0717$ & 2.92 & $\mathbf{1 4 6 0}$ & $\mathbf{1 0 0 0}$ & $-\mathbf{0 . 1 0 0}$ & 1631 \\
11944 & S09 L100 & $140101-0113$ & 2.68 & $\mathbf{1 5 6 0}$ & $\mathbf{8 3 0 0}$ & $\mathbf{- 0 . 1 3 5}$ & 1830 \\
11967 & S13 L114 & $140128-0209$ & 6.13 & $\mathbf{1 5 8 0}$ & $\mathbf{1 7 0 0}$ & $-\mathbf{0 . 1 7 6}^{*}$ & 1088 \\
12192 & S12 L248 & $141017-1030$ & $\mathbf{1 9 . 5 9}$ & $\mathbf{2 7 4 0}$ & 580 & $-\mathbf{0 . 2 1 9}$ & 677 \\
\hline
\end{tabular}

Notes: The parameter satisfying the criterion of SARs is indicated in boldface type. The asterisk $(*)$ after the data of $\triangle \mathrm{TSI}$ indicates that the data is got from FY3C/SIM.

four parameters, we proposed a composite vector field index $\left(I_{c o m}\right)$, and the majority of the SARs had high $I_{\text {com }}$ exceeding 1.0.

In this paper, we first examine the number and characteristics of the SARs in Solar Cycle 24 with the emphasis on understanding the general activity level of the current cycle. Then, we extend our sample of SARs by including that in Solar Cycle 24, and examine if the vector magnetic field parameters extracted from the studies in Paper II are valid for the new SARs. We discuss the general trend of activity level of the current cycle in Sect. 2, study the characteristics of SARs in Cycle 24 in Sect. 3, and present our conclusions and discussions in the last section.

\section{General trend of activity level of current solar cycle}

We use the data of TSI, number of sunspots, X class X-ray flares and number of SARs to show the development of the current solar cycle. The data of TSI between November 17, 1978 and March 28, 2012 used was PMOD composite TSI (http://www.acrim.com, for details see Fröhlich 2006). The data of TSI between March 29, 2012 and November 30, 2014 used was observed by Total Irradiance Monitor on the Solar Radiation and Climate Experiment (SORCE/TIM, http://lasp.colorado.edu). Other data were taken from National Geophysical Data Center (ftp://ftp.ngdc.noaa.gov) and Space Weather Prediction Center (http://www.swpc.noaa.gov). The coronal mass ejection (CME) velocity in Table 1 is the linear speed obtained from the LASCO CME catalog (http://cdaw.gsfc.nasa.gov). In order to make the two part TSI data from different instrument consistent, a linear fit method is used between the PMOD composite and SORCE/TIM data from February 25, 2003 to March 28, 2012 firstly. Then, we use the result of the linear fit to revise the data of SORCE/TIM from March 29, 2012 to November 30, 2014.

According to the criterion in Paper I, we identify five SARs in the current cycle, which are listed in Table 1. Because the SORCE/TIM data has a gap when NOAA 11944 and 11967 was on the solar disk, we use the data of Solar Irradiation Monitor on Fengyun 3C satellite (FY-3C/SIM, http://satellite.cma.gov.cn) to calculate the shortterm change of TSI caused by the two SARs, respectively. Fig. 1 shows the distribution of TSI (blue periods and curves), number of sunspots (black curves), X class X-ray flares (red histogram) and SARs (green histogram) in cycles 21-24. It can be seen that all of the above parameters decrease from cycle 21 to 24 . The monthly STSI decreased $0.09 \%$ from the maximum of cycle 23 to the minima between cycle 23 and 24 , while it decreased only about $0.07 \%$ in other cycles. The minimum of monthly SSN between cycle 23 and 24 is only 1.7. The Cycle 24 has passed its maximum from the distribution of monthly SSN (thick black curves), and the maximum monthly SSN is 81.9 which came in April 2014 and is only $67.8 \%, 51.6 \%$ and $49.8 \%$ of that of cycles 21-23, respectively. Moreover, there are only five SARs and 45 X class flares in Cycle 24 until August 31, 2015. 


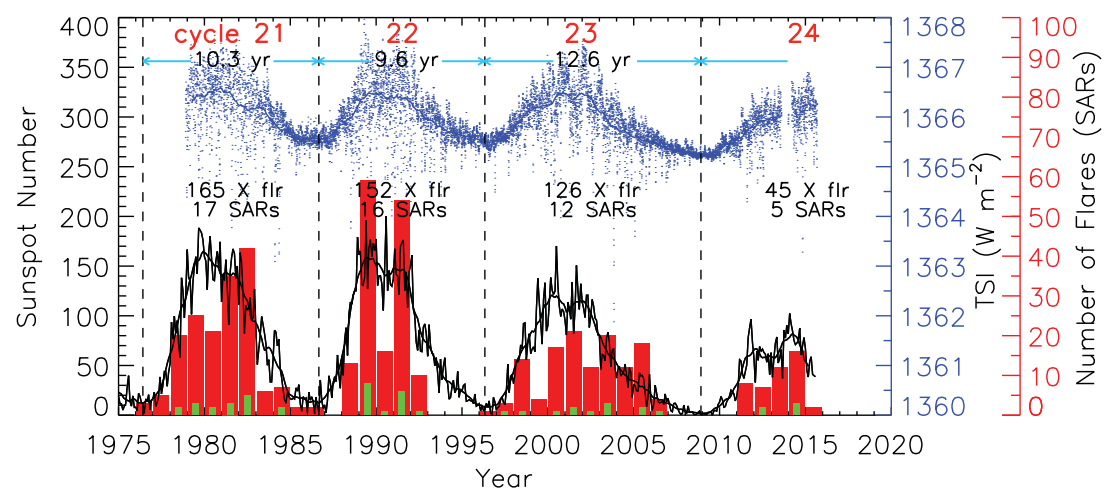

Figure 1. Distribution of TSI, number of sunspots, X class X-ray flares and SARs. Daily TSI and monthly STSI are represented by blue periods and curves, respectively. Monthly sunspot numbers and monthly SSN are represented by thin black and thick black curves, respectively. Number of X class flares and SARs are displayed by histograms in red and green colors, respectively. ' $\mathrm{X}$ flr' is the abbreviations of ' $\mathrm{X}$ class flare'. The vertical dashed indicates the position of the solar minimum of each solar cycle.

The duration(12.6 years) and the descending phase (8.7 years) of cycle 23 , and the ascending phase of Cycle 24 (5.3 years) are very long. From above analysis, it can be seen that we are probably facing the start of a new grand minimum (Feynman \& Ruzmaikin 2011; Solanki \& Krivova 2011; Zolotova \& Ponyavin 2014). Jin \& Wang (2014) suggested a similarity of cycles 11 and 23 by using a technique of normalized solar cycles. This similarity holds the prediction that Cycle 24 would be similar to cycle 12 , having a mini-maximum after the grand minima.

\section{Characteristics of SARs in Solar Cycle 24}

In Paper II, we used the vector magnetograms of the Solar Magnetic Field Telescope at Huairou Solar Observing Station and selected four magnetic field parameters to describe the vector field characteristics of SARs and FARs in cycles 22 and 23 . The four parameters were: 1 ) the net magnetic flux; 2) the total photospheric free magnetic energy density; 3) the length of the magnetic neutral line with steep horizontal magnetic gradient $(\geqslant 300 \mathrm{G}$ $\mathrm{Mm}^{-1}$ ); and 4) the area with strong magnetic shear (shear angle $\geqslant 80^{\circ}$ ). According to the statistical results of the four parameters of SARs and FARs, we proposed a composite vector field index ( $I_{\text {com }}$, for details see the Eq. (9) in Paper II). It was found that for the the majority of SARs $I_{c o m} \geqslant 1.0$.

In this paper, we use the vector magnetograms of the Helioseismic and Magnetic Imager onboard the Solar Dynamics Observatory (SDO/HMI, Schou et al. 2012), which have a spatial resolution of $1^{\prime \prime}$ and a time resolution of $12 \mathrm{~min}$, to calculate the above four parameter and the $I_{\text {com }}$ of SARs in Solar Cycle 24 according to the Eq. (9) in Paper II. The vector magnetograms used are the Space weather HMI Active Region Patch (SHARP) data (http://jsoc.stanford.edu), which had been removed the $180^{\circ}$ ambiguity and transformed from the image plane into the heliographic coordinate system by the HMI team. To make our result more reliable, we only select the magnetograms closing to the central meridian ( \pm 2.5 days). Because there are no FARs according to the definition of FAR in Paper II until August 31, 2015 in the current cycle, we took a lower area threshold of FAR for the current cycle. If an AR covered an area larger than $800 \mu \mathrm{h}$ and did not produce any flare higher than the M1.0 class, we refer to it as an FAR. There are two FARs which are studied to compare with the SARs. 
Table 2. The vector magnetic field parameters of SARs in Solar Cycle 24

\begin{tabular}{ccccccc}
\hline NOAA & $\begin{array}{c}\left|\Phi_{\text {net }}\right| \\
\left(10^{22} \mathrm{Mx}\right)\end{array}$ & $\begin{array}{c}\mathrm{E}_{\text {free }} \\
\left(10^{24} \mathrm{erg} \mathrm{cm}^{-1}\right)\end{array}$ & $\begin{array}{c}\mathrm{L}_{N L} \\
(\mathrm{Mm})\end{array}$ & $\begin{array}{c}\mathrm{A}_{\Psi}\left(\Psi \geqslant 80^{\circ}\right) \\
\left(\mathrm{Mm}^{2}\right)\end{array}$ & $\mathrm{I}_{\text {com }}$ \\
\hline \multirow{4}{*}{ SARs } & 11429 & 0.18 & 0.68 & $\mathbf{5 3 . 1 4}$ & $\mathbf{1 5 4 . 7 5}$ & $\mathbf{1 . 0 2}$ \\
& 11520 & 0.22 & 0.73 & 16.67 & $\mathbf{4 0 4 . 9 6}$ & $\mathbf{1 . 3 5}$ \\
& 11967 & $\mathbf{1 . 2 1}$ & 0.30 & 25.33 & $\mathbf{1 4 4 . 3 3}$ & $\mathbf{1 . 0 4}$ \\
& $\mathbf{1 . 5 1}$ & $\mathbf{1 . 4 3}$ & $\mathbf{8 7 . 6 5}$ & $\mathbf{3 1 6 . 4 7}$ & $\mathbf{2 . 3 2}$ \\
\hline FARs & 12192 & $\mathbf{1 . 2 6}$ & 0.57 & $\mathbf{3 5 . 0 7}$ & $\mathbf{1 0 3 . 2 2}$ & $\mathbf{1 . 1 0}$ \\
& 12108 & 0.09 & 0.04 & 6.45 & 12.59 & 0.13 \\
& 12109 & 0.46 & 0.05 & 3.93 & 24.33 & 0.26
\end{tabular}

Notes: The parameter value that is larger than the threshold value of SARs in paper II is indicated in boldface type.

The values of above four parameters and $I_{\text {com }}$ of five SARs and two FARs in Cycle 24 are listed in Table 2. Fig. 2 shows the relationship between $I_{\text {com }}$ and the soft X-ray flare index $\left(I_{\text {flare }}\right)$ of $19 \mathrm{SARs}$ and $10 \mathrm{FARs}$ in cycles 22-24. There appears a good linear correlation between the two parameters of SARs, and the linear correlation coefficient is 0.69. A t-test is used to examine the confidence level, and the confidence level of correlation is larger than 99\%. All the SARs (red circle) in Cycle 24 have an $I_{\text {com }}>1.0$, which consists with the characteristics of SARs (red plus) in cycles 22 and 23. However, the $I_{\text {com }}$ of all the FARs (blue circle) are lower than 1.0. If we decrease the threshold of the $I_{\text {com }}$ to 0.9 (see the green dash-dotted line in Fig. 2), all the SARs and FARs in the three cycles would be easily distinguished except for one FAR. Comparing with SARs in cycles 22 and 23, the $I_{\text {flare }}$ and the $I_{\text {com }}$ of SARs in Cycle 24 are all relatively lower. For Cycle 24, two SARs have a $I_{\text {flare }}<3.0$. However, in cycles 21-23, the $I_{\text {flare }}$ of all SARs are higher than 6.0 (see Table A.1 of Paper I). The velocities of CMEs associated with SARs in Cycle 24 are relatively lower. There are two SARs did not produce CMEs with velocity larger than $1500 \mathrm{~km} \mathrm{~s}^{-1}$. However, almost all the SARs are likely to produce CMEs with velocity larger than $1500 \mathrm{~km} \mathrm{~s}^{-1}$ in cycle 23 (Chen et al. 2011). This indicates again that the activity level of Cycle 24 is much weaker in comparing with the three preceding cycles in term of SARs. From Table 2, we see that all the SARs in Cycle 24 have a larger area with strong magnetic shear which is larger than $100 \mathrm{Mm}^{2}$. However, most of them have a lower total photospheric free magnetic energy density which is lower than $1.0 \times 10^{24}$ erg $\mathrm{cm}^{-1}$. The magnetic free energy in the photosphere seems to be the most decisive parameter to highlight the major solar activity (Wang et al. 1996; Leka \& Barnes 2007; Chen et al. 2011). Some SARs are notable, for example, NOAA 11967 had a highest $I_{\text {com }}$, but did not produce any flare higher than the X1.0 class; while NOAA 12192 had the largest $I_{\text {flare }}$ in the current cycle and had the largest sunspot area since December 1990, but was clearly CME-poor; among tens of major flares, only an M4.0 flare was associated with a CME.

Many authors found that CMEs initiation was associated with the large-scale coronal connectivity (Wang et al. 2006; Wang et al. 2007; Zhang et al. 2007; Zhou et al. 2007; Schrijver \& Title 2011; Schrijver et al. 2013). Recently, Wang et al. (2015) described a new type of magnetic complex: AR cluster. An AR cluster was referred to as the close connection of two or more ARs. They studied three AR clusters, and found that CMEs initiations in the clusters were often tied to the instability of the magnetic structures connecting two partner ARs and the magnetic interaction in the whole AR cluster. It can be seen that magnetic connectivity between ARs is an important ingredient to major solar activity, particularly the high speed CMEs. In this paper, we browsed the SDO/AIA $171 \AA$ images of these five SARs, and found that all SARs except NOAA 12192 were magnetically connected with other ARs. The overall connectivity of NOAA 11429 are 


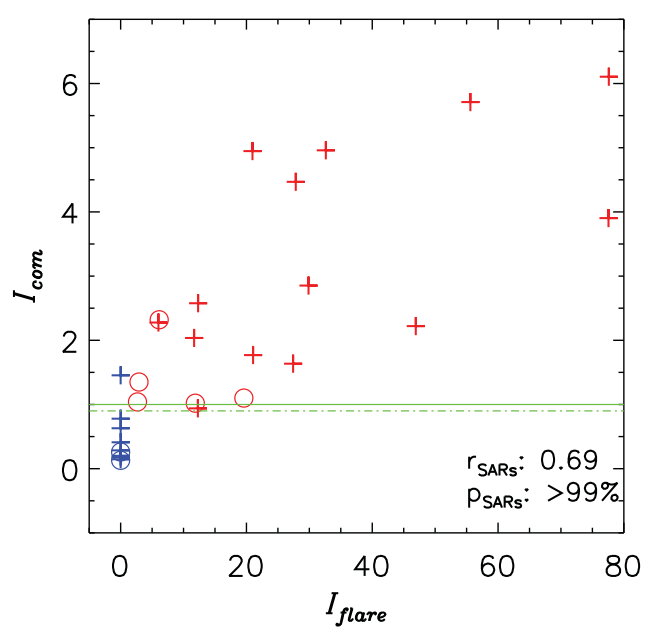

Figure 2. Relationship between the composite vector field index $I_{\text {com }}$ and the soft X-ray flare index of ARs. Each plus represents an SAR (red color) or an FAR (blue color) in solar cycles 21-23, and each open circle represents an SAR (red color) or an FAR (blue color) in Cycle 24, respectively. The green line represents the threshhold value distinguishing the SARs and FARs in Paper II, and the green dash-dotted line represents the composite index $I_{\text {com }}$ equal to 0.9 .

shown in Fig. 3. We can see that NOAA 11429 connects with NOAA 11430 by loops (thin arrows) and connects with the flocculus in the southern hemisphere by trans-equatorial loops (thick arrow). The eruption of the loops connecting the negative polarity of NOAA 11429 and the positive polarity of NOAA 11430 was associated with an X1.3 flare and a CME with a speed of $1825 \mathrm{~km} \mathrm{~s}^{-1}$ on March 7, 2012.

\section{Conclusions and discussions}

According to the criterion established based on the previous studies of SARs (see Paper I), there have been only five SARs in Solar Cycle 24. In this work we studied the distribution of TSI, numbers of sunspots and X class of X-ray flares, as well as the SARs, of cycles 21-24, and analyzed the vector magnetic characteristics of five SARs and two FARs in Cycle 24. the following results were obtained.

(1) The solar activity level have a monotonic decreasing trend from cycle 21 to 24 , and the current cycle has a mini-maximum.

(2) The magnetic characteristics of SARs and FARs in the current cycle follow the characteristics of that in cycles 22 and 23 . However, the $I_{\text {com }}$ of them is relatively smaller and only three SARs were associated with CMEs of velocity larger than $1500 \mathrm{~km} \mathrm{~s}^{-1}$. $I_{\text {com }}$ is linearly proportional to the $I_{\text {flare }}$ of SARs.

(3) Most SARs show magnetic connectivity with other spacely-closed active regions.

The solar activity level of the current cycle is so low, and we are possibly facing the start of a new grand minimum (Feynman \& Ruzmaikin 2011; Solanki \& Krivova 2011; Zolotova \& Ponyavin 2014; Jin \& Wang 2014). The low $I_{\text {flare }}, I_{\text {com }}$ and CME velocity of SARs may be related to the peculiarity of dynamo action which causes a lower sunspot number, fewer X class X-ray flares and SARs. The $I_{c o m}$ may be a good parameter to describe the activity level of SARs, and can be used to quantify and prediction the super activity events. The magnetic connectivity between SARs and other space-closed active regions was revealed by the successful SDO/AIA facilities and might be common for SARs in previous solar cycles. 


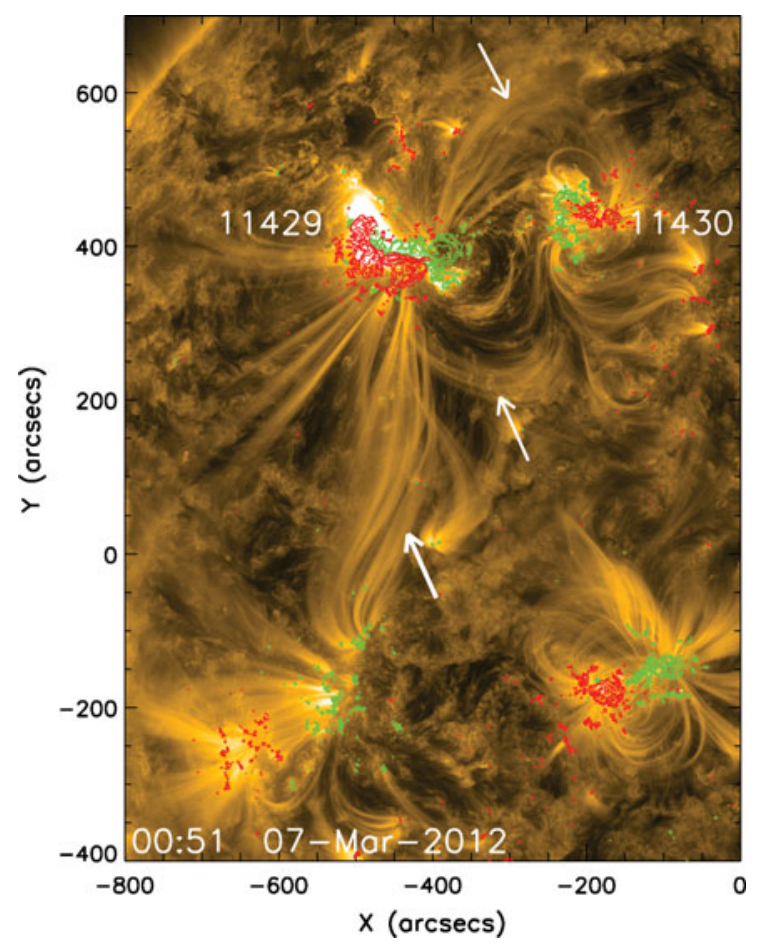

Figure 3. Overall connectivity of NOAA 11429 shown by AIA $171 \AA$ images superposed by the magnetic flux density with green (red) lines for positive (negative) polarity and contours of $\pm 200,500,1000,1500 \mathrm{G}$. The two thin white arrows indicate the inter-connecting loops between two ARs and the thick white arrows indicates the trans-equatorial loop.

\section{Acknowledgements}

The research is supported by the National Natural Science Foundation of China (41404150, 41327802, 41204104 and 11573038) and the collaborating research program of Key Laboratory of Solar Activity of Chinese Academy of Sciences (KLSA201410).

\section{References}

Chen, A. Q., Wang, J. X., \& Li, J. W., et al. 2011, A\&A, 534, A47

Chen, A. Q. \& Wang, J. X. 2012, A\& A, 543, A49

Feynman, J. \& Ruzmaikin, A. 2011, Solar Phys., 272, 351

Fröhlich, C. 2006, Space Sci. Revs, 125, 53

Jin, C. L. \& Wang, J. X. 2014, J. Geophys. Res., 119, 11

Leka, K. D. \& Barnes, G. 2007, ApJ 656, 1173,

Schrijver, C. J. \& Title, A. M. 2011, J. Geophys. Res., 116, A04108

Schrijver, C. J., Title, A. M., \& Yeates, A. R., et al. 2013, J. Geophys. Res., 773, 93

Schou, J., Scherrer, P. H., \& Bush, R. I., et al. 2012, Solar Phys., 275, 229

Solanki, S. K. \& Krivova, N. A. 2011, Science, 334, 916

Wang, J. X., Shi, Z. X., Wang, H. N., \& Lü, Y. P. 1996, ApJ, 456, 861

Wang, J. X., Zhou, G. P., \& Wen, Y. Y., et al. 2006, Chin J Astron Astrophys, 6, 247

Wang, J. X., Zhang, Y. Z., \& Zhou, G. P., et al. 2007, Solar Phys., 244, 75

Wang, J. X., Zhang, Y. Z., \& He, H., et al. 2015, Sci China-Phys Mech Astron, 58, 599601

Zhang, Y. Z., Wang, J. X., \& Attrill, G. D. R., et al. 2007, Solar. Phys., 241, 329

Zhou, G. P., Wang. J. X. \& Wang, Y. M., et al. 2007, Solar. Phys., 244, 13

Zolotova, N. V., \& Ponyavin, D. I. 2014, J. Geophys. Res., 119, 3281 